\title{
Estimativas de (Co)Variâncias entre Características de Reprodução e de Crescimento em Fêmeas de um Rebanho Nelore ${ }^{1}$
}

\author{
Maria Eugênia Zerlotti Mercadante ${ }^{2}$, Raysildo Barbosa Lôbo ${ }^{3}$, Henrique Nunes de Oliveira ${ }^{4}$
}

RESUMO - Informações de 7986 nascimentos de animais da raça Nelore, ocorridos entre 1960 e 1993, provenientes de um rebanho do Estado de São Paulo, foram usadas para estudar componentes de (co)variâncias, herdabilidades e correlações genéticas de peso ao desmame (P240), peso a um ano de idade (P365), idade ao primeiro parto (IPP), primeiro intervalo de partos (IEP1), eficiência reprodutiva (ER), anos de permanência da matriz no rebanho (LONG) e peso ao desmame do primeiro bezerro da matriz (P240B). As análises foram realizadas utilizando-se o software MTDFREML, estimando-se os componentes de (co)variâncias por máxima verossimilhança restrita, assumindo modelo animal. As estimativas de herdabilidade apresentaram resultados similares entre as diferentes análises, sendo mais altas $(0,26$ a 0,35) para P240, P365 e IPP e mais baixas $(0,08$ a 0,26) para IEP1, ER, LONG e P240B. De modo geral, as estimativas de correlação genética e fenotípica entre crescimento e reprodução foram baixas e entre as características de reprodução, mais altas e de sentido favorável. Algumas correlações entre o efeito genético materno das características de crescimento e o direto das características de reprodução foram de média magnitude e de sentido desfavorável, sugerindo antagonismo genético entre produção de leite e reprodução.

Palavras-chave: crescimento, fêmeas zebuínas, parâmetros genéticos, raça Nelore, reprodução

\section{Estimates of (Co) Variances among Reproductive and Growth Traits in Female Nellore Cattle}

\begin{abstract}
Informations of 7986 births of animals from a Nellore cattle herd, from 1960 to 1993, were used to estimate (co) variance components, heritabilities and genetic correlations of weaning weight (W240), yearling weight (W365), age at first calving (AFC), first calving interval (CI1), reproductive efficiency (RE), longevity (LONG), calculated as years of permanence of cow in herd and weaning weight of the first calf of cow (W240C). The analysis were performed using the MTDFREML software, estimating the (co) variance components by restricted maximum likelihood, assuming an animal model. Estimates of heritability showed similar results among the different analyses, being higher (0.26 to 0.35) for W240, W365 and AFC and lower (0.08 to 0.26) for CI1, RE, LONG and W240C. In general, the estimates of genetic and phenotypic correlations among growth and reproductive traits were low and among the reproductive traits were high and favorable. Some correlations between maternal genetic effect of the growth and the direct genetic effect of the reproductive traits were of average magnitude and unfavorable, suggesting genetic antagonism between milk production and reproduction.
\end{abstract}

Key Words: genetic parameters, growth, Nellore breed, reproduction, zebu female

\section{Introdução}

As características de reprodução são de suma importância em qualquer exploração pecuária, porém são difíceis de serem mensuradas e interpretadas, já que são influenciadas por interações entre bezerro, touro e vaca. Além disso, apresentam baixos coeficientes de herdabilidade. Estudos indicam que esses coeficientes são muito baixos em condições temperadas e pouco mais altos para animais Bos indicus e mestiços em condições tropicais, sugerindo que há maior oportunidade de seleção para fertilidade em Bos indicus que em Bos taurus (MORRIS, 1990). Por outro lado, as características de crescimento são fáceis de se medir e interpretar e apresentam coeficientes de herdabilidade mais altos, respondendo bem à seleção.

As relações entre os vários estádios do crescimento são bem documentadas (MOHIUDDIN, 1993; MERCADANTE et al., 1995); para características de reprodução, o número de estudos não é tão grande (KOOTS et al., 1994a,b). Há algumas evidências de antagonismo genético entre crescimento e reprodução em ambiente desfavorável (MARIANTE e ZANCANER, 1985; BARBOSA, 1991), contudo, há

\footnotetext{
${ }^{1}$ Parte da Dissertação de Mestrado do primeiro autor - FMRP - USP. Apoio financeiro: CAPES e FINEP.

${ }^{2}$ E. E. Zootecnia de Sertãozinho - IZ/SAA-SP - Caixa Postal, 63 - Sertãozinho, SP. E.mail: meugenia@imagenet.com.br

${ }^{3}$ Depto. Genética - Faculdade Medicina de Ribeirão Preto - USP - Av. Bandeirantes, 3900 Ribeirão Preto-SP. E.mail: rayblobo @ genbov.fmrp.usp.br

${ }^{4}$ Professor da UNESP/Botucatu - Departamento de Melhoramento e Produção Animal - Lageado. Cx. Postal 560 - CEP: $18168-000$ - Botucatu, SP. E.mail: hnunes@fca.unesp.br
} 
estudos indicando que estas características estão pouco relacionadas (SMITH e BRINKS, 1989), ou mesmo favoravelmente relacionadas, com algumas características, como evidenciado nos trabalhos de BARBOSA (1991) e MEYER et al. (1991). Entretanto, existem lacunas na literatura, em particular no que diz respeito às relações fenotípicas e genéticas entre aspectos de reprodução, lactação e eficiência da produção "vaca-bezerro".

Quando o nível de nutrição é inadequado, as vacas tendem a manter o nível de produção de leite de acordo com seu potencial genético, às expensas das reservas corporais, afetando a eficiência reprodutiva. Confirmando isto, DAVIS et al. (1993) reportaram resultados obtidos em um experimento de seleção divergente para taxa de prenhez em bovinos de corte, em que a seleção de touros com base em DEPs (diferença esperada na progênie) para maiores taxas de prenhez de suas filhas levou ao aumento da fertilidade, sem efeitos adversos sobre o crescimento pós-desmame da progênie, porém o peso ao desmame dos bezerros da linha selecionada para alta taxa de prenhez foi significativamente menor que os da linha selecionada para baixa taxa de prenhez.

Os objetivos do presente trabalho foram: a) calcular estimativas de variância e herdabilidade para características de reprodução (idade ao primeiro parto, primeiro intervalo de partos, eficiência reprodutiva e tempo de permanência da matriz no rebanho) e produção (peso ao desmame do primeiro bezerro da matriz); e b) avaliar as estimativas de covariâncias e correlações genéticas entre as características de reprodução e os efeitos genéticos direto e materno dos pesos ao desmame e a um ano de idade, bem como as correlações fenotípicas entre as características estudadas, em fêmeas de um rebanho da raça Nelore.

\section{Material e Métodos}

Os dados são referentes a 7986 nascimentos de animais da raça Nelore ocorridos entre 1960 e 1993, na Fazenda Bonsucesso, localizada no município de Guararapes-SP, participante do "Programa de Melhoramento Genético da Raça Nelore", coordenado pelo Departamento de Genética da Faculdade de Medicina de Ribeirão Preto desde 1988. A fazenda está localizada, aproximadamente, a $21^{\circ} 12^{\prime}$ de latitude sul e $50^{\circ}$ 26' de longitude oeste, com estações chuvosa e seca bem definidas. Os animais foram criados em pasto e o desmame ocorreu entre 7 e 8 meses de idade. Uma estação de monta fixa foi implementada e, a partir de
1980, o acasalamento concentrou-se nos meses de outubro, novembro e dezembro.

As novilhas foram colocadas em reprodução por volta dos 24 meses de idade. O critério de seleção das fêmeas baseou-se no peso e na fertilidade, sendo retidas no rebanho aquelas que apresentaram peso superior à média das contemporâneas e filhas de vacas férteis. As fêmeas que não emprenharam durante a estação de monta ou desmamaram bezerro muito leve em um ano foram descartadas, se repetissem no ano seguinte. Foram selecionados os machos com maior peso aos 18 e 24 meses de idade, sendo usados como reprodutores na própria fazenda.

As características de crescimento estudadas foram os pesos ao desmame, padronizado para 240 dias (P240), e a um ano de idade, padronizado para 365 dias (P365). Com relação à reprodução, foram estudadas as características idade ao primeiro parto (IPP), primeiro intervalo de partos (IEP1), eficiência reprodutiva (ER) e anos de permanência da matriz no rebanho (LONG). O peso ao desmame do primeiro bezerro da matriz (P240B) foi considerado característica de produção.

Os limites considerados foram 26 a 47 meses para IPP e 300 a 790 dias para IEP1. A ER foi calculada como proposto por BEZERRA e DUARTE (1980): $[(274(\mathrm{n}-1)) / \Sigma \text { iep x }(24 / \mathrm{ipp})]^{1 / 2}$, em que n é o número de partos; $\Sigma$ iep, a somatória dos (n-1) intervalos de partos; e ipp, a idade ao primeiro parto, sendo então considerados todos os partos da matriz (25\% das matrizes com um só parto e o restante variando de 2 a 14 partos). A LONG só foi calculada para as matrizes que possuíam a informação da data de nascimento e nascidas até 1982, já que as nascidas em idades posteriores não tiveram a mesma oportunidade de parir e de permanecer tempo suficiente no rebanho.

As estações de nascimento dos animais foram agrupadas em quatro, dentro de ano de nascimento: janeiro, fevereiro e março (estação 1); abril, maio e junho (estação 2); julho, agosto e setembro (estação 3); e outubro, novembro e dezembro (estação 4). A idade da mãe ao parto foi dividida em seis classes: menor ou igual a três anos, quatro anos, cinco anos, seis anos, sete a dez anos e maior que dez anos. Todas as informações disponíveis sobre a genealogia dos animais foram coletadas.

A estrutura dos dados, a média geral e o desviopadrão das características estudadas são mostrados na Tabela 1.

Os componentes de (co)variâncias foram estimados por máxima verossimilhança restrita não-derivativa, usando o software MTDFREML (BOLDMAN et al., 1993), assumindo modelo animal. O modelo animal é mais vantajoso para características de menor herdabilidade como as características de reprodução das 
Tabela 1 - Estrutura dos dados, médias e desvio-padrão das características

Table 1 - Data structure, means and standard deviation of the traits

\begin{tabular}{|c|c|c|c|c|c|c|c|}
\hline & $\begin{array}{c}\mathrm{P} 240^{\mathrm{a}} \\
W 240 \\
(\mathrm{~kg})\end{array}$ & $\begin{array}{c}\mathrm{P} 365^{\mathrm{a}} \\
W 365 \\
(\mathrm{~kg})\end{array}$ & $\begin{array}{c}\text { IPP } \\
\text { AFC } \\
\text { (meses/ } \\
\text { months) }\end{array}$ & $\begin{array}{c}\text { IEP1 } \\
\text { IEP1 } \\
\text { (dias/ } \\
\text { days) }\end{array}$ & $\begin{array}{l}\mathrm{ER} \\
R E\end{array}$ & $\begin{array}{l}\text { LONG } \\
\text { LONG } \\
\text { (meses/ } \\
\text { months) }\end{array}$ & $\begin{array}{c}\mathrm{P} 240 \mathrm{~B}^{\mathrm{b}} \\
P 240 B \\
(\mathrm{~kg})\end{array}$ \\
\hline № animais & 3237 & 3192 & 1217 & 958 & 979 & 693 & 1041 \\
\hline $\begin{array}{l}\mathrm{N}-\text { animais em } \mathrm{A}^{-1} \\
N \text {. animals in } A^{-1}\end{array}$ & 4487 & 4487 & 1591 & 1591 & 1591 & 1591 & 1591 \\
\hline $\begin{array}{l}\text { № touros } \\
N \text {. sires }\end{array}$ & 105 & 103 & 87 & 77 & 77 & 54 & 63 \\
\hline $\begin{array}{l}\text { № ano-estac. nasc. } \\
\text { N. year-season birth }\end{array}$ & 109 & 108 & 90 & - & 88 & 68 & 93 \\
\hline $\begin{array}{l}\text { № ano-estac. parto } \\
N \text {. year-season calving }\end{array}$ & - & - & - & 95 & - & - & - \\
\hline $\begin{array}{l}\text { Média geral } \\
\text { Overall mean }\end{array}$ & 174,0 & 187,0 & 38,3 & 471,0 & 0,64 & 104,8 & 177,0 \\
\hline $\begin{array}{l}\text { Desvio-padrão } \\
\text { Standard deviation }\end{array}$ & 24,89 & 26,35 & 3,51 & 113,97 & 0,05 & 40,15 & 28,27 \\
\hline
\end{tabular}

a Fêmeas, ${ }^{b}$ machos e fêmeas.

P240, peso ao desmame; P365, peso aos 365 dias; IPP, idade ao primeiro parto; IEP1, primeiro intervalo de partos; ER, eficiência reprodutiva; LONG, anos de permanência da matriz no rebanho; P240B, peso ao desmame do primeiro bezerro da matriz.

a Females, ${ }^{b}$ males andfemales.

W240, weaning weight; W365, yearling weight; AFC, age of first calving; C11, first calving interval; RE, reproductive efficiency, LONG, longevity; W240C, weaning weight of first calf of cow.

fêmeas. A implementação de análise multivariada adiciona valores para estas características, especialmente quando são correlacionadas com características de maior herdabilidade e menos dispendiosas para mensurar, como as de crescimento (MEYER et al., 1991).

Para as características de crescimento (P240 e P365), o modelo incluiu efeitos genéticos direto e materno e efeito de ambiente permanente como aleatórios e os efeitos de ano-estação de nascimento e idade da mãe ao parto como fixos. O modelo para as características de reprodução incluiu efeito genético direto da matriz como aleatório e os efeitos de anoestação de nascimento para IPP, ER e LONG e anoestação de parição para IEP1 como fixos. Para P240B o modelo incluiu efeito genético direto da matrize efeito do pai do bezerro, como aleatórios e não-correlacionados, e os efeitos fixos de sexo e ano-estação de nascimento do bezerro. Nesse caso, o efeito do pai do bezerro explica metade da variância genética aditiva do P240B, e o efeito genético direto da matriz, todo o efeito genético materno dela (que incide sobre o P240B como ambiente) mais um quarto da variância genética aditiva da característica em questão, além das possíveis interações entre estes dois efeitos.

Inicialmente as características foram analisadas de forma isolada e vários modelos foram testados. Posteriormente, foram realizadas análises bivariadas. $\mathrm{O}$ critério de convergência considerado foi de $10^{-9} \mathrm{e}$, a cada convergência, o programa era reiniciado, usando como valores iniciais aqueles obtidos na análise anterior.

De forma matricial, o modelo pode ser descrito como:

$$
\mathrm{y}=\mathrm{X} \beta+\mathrm{Z}_{1} \mathrm{~g}+\mathrm{Z}_{2} \mathrm{~m}+\mathrm{Z}_{3} \mathrm{p}+\mathrm{e}
$$

em que y é o vetor de observações (P240, P365, IPP, IEP1, LONG e P240B); X, a matriz de incidência dos efeitos fixos (ano-estação de nascimento ou parto, classe de idade da mãe ao parto e sexo do bezerro, dependendo da característica); $\beta$, o vetor dos efeitos fixos; $Z_{1}$, a matriz de incidência dos efeitos genéticos direto (P240, P365, IPP, IEP1, LONG e P240B); g, o vetor de efeitos genéticos direto; $\mathrm{Z}_{2}$, a matriz de incidência dos efeitos genéticos materno (P240 e P365); m, o vetor dos efeitos genéticos materno; $Z_{3}$, a matriz de incidência dos efeitos de ambiente permanente (P240 e $\mathrm{P} 365$ ) ou efeito do pai do bezerro (P240B); $\mathrm{p}$, o vetor dos efeitos de ambiente permanente; e $\varepsilon$, o vetor dos erros aleatórios residuais associados às observações.

\section{Resultados e Discussão}

\section{Componentes de (co)variâncias e estimativas de herdabilidade}

As estimativas de (co)variâncias genéticas (direta e materna) e fenotípica, herdabilidades e correlações genéticas e fenotípicas entre as características de 
crescimento e reprodução são apresentadas na Tabela 2.

Análises univariadas das características P240 e

P365 foram apresentadas e discutidas anteriormente por MERCADANTE e LÔBO (1997). Os valores das estimativas de variância genética direta $\left(\sigma^{2}{ }_{\mathrm{a}}\right)$, genética materna $\left(\sigma_{m}^{2}\right)$ e fenotípica $\left(\sigma_{p}^{2}\right)$ para P240 e P365 variaram entre as análises, ocorrendo maior mudança, quando analisadas com LONG, principalmente nos componentes do efeito materno (genético e de ambiente permanente).

A magnitude da estimativa de herdabilidade do efeito genético direto $\left(\mathrm{h}^{2}{ }_{\mathrm{a}}\right)$, obtida para P240, foi similar àquelas relatadas na literatura para bovinos de origem indiana, enquanto a estimativa de herdabilidade do efeito genético materno $\left(\mathrm{h}^{2}{ }_{\mathrm{m}}\right)$ foi inferior, especialmente quando confrontada com aquelas obtidas em bovinos da raça Nelore no Brasil (MERCADANTE et al., 1995). Para P365, a estimativa de $\mathrm{h}^{2}$ a foi de maior magnitude do que as reportadas na literatura para as raças zebuínas e, em geral, ocorreu o contrário para a estimativa de $\mathrm{h}^{2}{ }_{\mathrm{m}}$. São poucos os estudos de $\mathrm{P} 365$ que incluem componentes do efeito materno em animais zebuínos, de qualquer forma parece mais lógico que esta característica mostre menores valores para estes componentes.

Para a característica IPP, as estimativas de $\sigma^{2}{ }_{\text {a }}$ $\mathrm{e}^{2}{ }_{\mathrm{a}}$ foram muito próximas nas duas análises, sendo esta última de maior magnitude que as obtidas em estudos com animais de raças européias (KOOTS et al., 1994a) e animais zebuínos usando modelo animal (HAILE-MARIAM e KASSA-MERSHA, 1994; OLIVEIRA, 1995), concordando com o valor obtido por LÔBO (1998). As estimativas de $\sigma^{2}{ }_{\mathrm{a}}$ para IEP1 diferiram também muito pouco entre as análises com as características de crescimento e, consequientemente, os valores para $h^{2}$ foram também muito próximos. Estimativas de $\mathrm{h}^{2}{ }_{\mathrm{a}}$ de baixa magnitude para esta característica ou características afins, como data do parto na estação de nascimento, taxa de prenhez, entre outras, são reportadas em vários estudos (MEYER et al., 1990; MEYER et al., 1991; REGE e FAMULA, 1993; e LÔBO, 1998). HAILEMARIAM e KASSA-MERSHA (1994) sugerem que menor estimativa de $\mathrm{h}^{2}$ a pode ser obtida no primeiro desempenho, já que as vacas jovens são mais vulneráveis ao estresse nutricional.

As estimativas de $\sigma^{2}{ }_{a} e^{2}{ }^{2}$ a para ER foram idênticas nas duas análises (uni e bivariada), sendo esta última de baixa magnitude $(0,13)$. Fórmulas que procuram englobar em uma só medida a produtividade total são atrativas e de fácil compreensão pelo produtor, porém devem ser usadas com certo cuidado, já que incorporam muitas características de diferentes herdabilidades e importância econômica. Em geral, a herdabilidade destas tende a ser controlada pela característica mais variável e de menor herdabilidade considerada na equação (NOTTER, 1995).

Os componentes de $\sigma^{2}{ }_{\mathrm{a}} \mathrm{e} \mathrm{h}^{2}$ a para LONG foram muito similares nas duas análises com P240 e P365. Quando análises bivariadas incluindo essa variável e as características de reprodução foram realizadas, foram obtidos valores $\mathrm{de}^{2}{ }_{\mathrm{a}}$ muito mais baixos para LONG (entre 0,02 e 0,08 ), fazendo com que a média das $\mathrm{h}^{2}$ obtidas em todas as análises realizadas fosse 0,08 (Tabela 3). Esta discrepância entre os valores de diferentes análises pode ser atribuída ao fato de o número de observações entre as características de crescimento (P240 e P365) e LONG ser muito diferente (Tabela 1), embora MEYER et al. (1991), analisando dados de crescimento e reprodução, em que o número de observações para as características de crescimento era muito maior ao das características de reprodução, encontraram valores muito similares entre as análises. Outra hipótese seria que as análises de LONG com as características de crescimento permitiram contornar os vieses decorrentes de seleção dos animais, uma vez que a amostra de P240 e P365 não é selecionada. O tempo de permanência da matriz no rebanhoé um indicador de sua habilidade reprodutiva, já que matrizes que apresentam falhas na reprodução tendem a ser descartadas mais cedo.

$\mathrm{O}$ peso ao desmame do primeiro bezerro da matriz (P240B) foi analisado somente com as características de reprodução, sendo a estimativa de $\mathrm{h}^{2}{ }_{\mathrm{a}}$ igual a 0,13 (Tabela 3). Essa característica reflete, entre outros aspectos, a capacidade da matriz de cuidar da cria e, principalmente, sua produção de leite. Valores de estimativas de $\mathrm{h}^{2}{ }_{\mathrm{a}}$ de maiores magnitudes são relatados por MEYER et al. (1994) e ALBUQUERQUE e FRIES (1997).

$\mathrm{Na}$ Tabela 3, estão as médias dos valores das estimativas de herdabilidade das características de reprodução e produção obtidos em análises uni e bivariadas. Com exceção do valor para LONG, já discutido anteriormente, todos os outros concordaram com os valores de $\mathrm{h}^{2}{ }_{\mathrm{a}}$ obtidos nas análises bivariadas envolvendo as características de crescimento.

\section{Covariâncias e correlações entre características de crescimento e reprodução}

As estimativas de correlações genéticas direta $\left(\mathrm{r}_{\mathrm{a} 1 \mathrm{a} 2}\right)$ e fenotípicas $\left(\mathrm{r}_{\mathrm{p} 1 \mathrm{p} 2}\right)$ entre P240 e P365 e as características de reprodução foram, de modo geral, de baixas magnitudes, algumas próximas de zero (Tabela 2). Os 
Rev. bras. zootec.

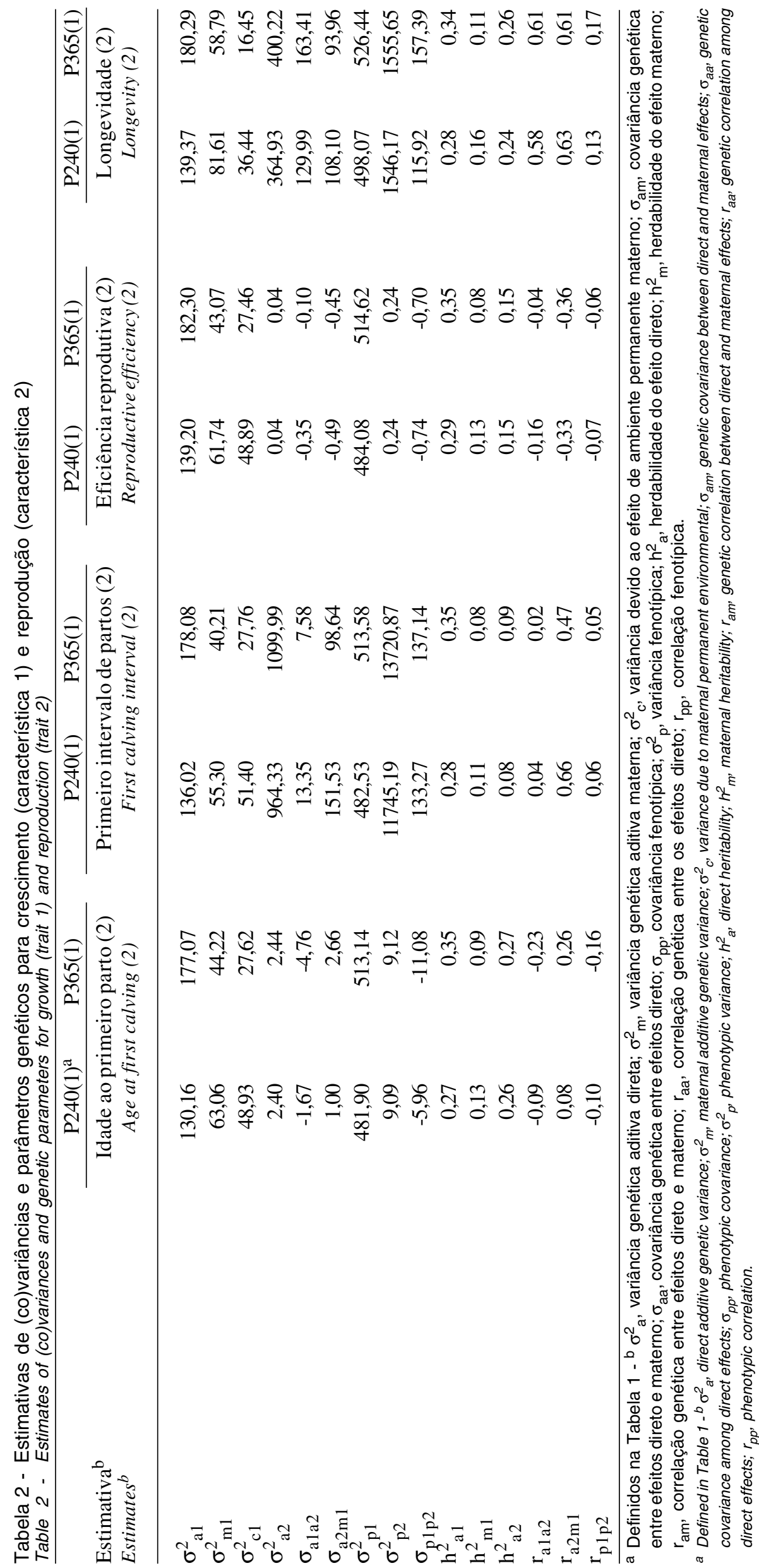


1002

Tabela 3 - Estimativas de herdabilidade ${ }^{a}$ (média das análises), correlação genética ${ }^{b}$ e fenotípica ${ }^{c}$ entre as características de reprodução

Table 3 - Estimates of heritability (means of analyses)a, and genetic $^{b}$ and phenotypic ${ }^{c}$ correlations between the reproductive traits

\begin{tabular}{lccccc}
\hline & IPP & IEP1 & ER & LONG & P240B \\
\hline IPP & $\mathbf{0 , 2 8}$ & $-0,06$ & $-0,44$ & $-0,12$ & 0,06 \\
IEP1 & 0,53 & $\mathbf{0 , 1 0}$ & $-0,56$ & 0,02 & 0,11 \\
ER & $-0,80$ & $-0,93$ & $\mathbf{0 , 1 6}$ & 0,23 & $-0,56$ \\
LONG & $-0,44$ & 0,07 & 0,68 & $\mathbf{0 , 0 8}$ & 0,04 \\
P240B & $-0,09$ & 0,60 & $-0,12$ & 0,16 & $\mathbf{0 , 1 3}$ \\
\hline
\end{tabular}

adiagonal, babaixo da diagonal, cacima da diagonal.

a diagonal, ${ }^{b}$ below diagonal, ${ }^{c}$ above diagonal.

valores obtidos na análise que incluiu LONG devem ser interpretados com certo cuidado, já que foi esta que forneceu valores de $\mathrm{h}^{2}{ }_{\mathrm{a}}$ para LONG bem discrepantes em relação às outras análises.

As estimativas de $\mathrm{r}_{\mathrm{a} 1 \mathrm{a} 2}$ entre P240 e P365 e a característica IPP foram baixas e de sentido favorável à seleção, enquanto as obtidas com a característica IEP1 foram também baixas (bem próximas de zero), porém de sentido desfavorável. A mesma tendência foi verificada nos valores de $\mathrm{r}_{\mathrm{p} 1 \mathrm{p} 2}$. Sabe-se que a idade à primeira cobrição e, conseqüentemente, a IPP é muito mais função do peso corporal do que da idade do animal, já que é prática comum em muitos rebanhos a adoção de peso mínimo para a entrada das fêmeas na reprodução. Tal fato pode provocar esta relação favorável entre as características de crescimento e IPP. MASCIOLI et al. (1999) relataram estimativas de correlação genética favorável entre peso a um ano e IPP $(-0,32)$ e entre peso a um ano e peso ao primeiro parto $(0,76)$.

A característica eficiência reprodutiva não mostrou relação com as características de crescimento, como pode ser observado pelos baixos valores, próximos de zero, das estimativas de $r_{\mathrm{a} 1 \mathrm{a} 2}$ e $\mathrm{r}_{\mathrm{p} 1 \mathrm{p} 2}$, contudo, o valor da estimativa de $\mathrm{r}_{\mathrm{a} 1 \mathrm{a} 2}$ entre P240 e ER igual a -0,16, apesar de muito baixo, sugere certa tendência antagônica entre as características.

Com relação à LONG com P240 e P365, as estimativas de $\mathrm{r}_{\mathrm{a} 1 \mathrm{a} 2}$ e $\mathrm{r}_{\mathrm{p} 1 \mathrm{p} 2}$ foram de maiores magnitudes quando comparadas com as demais. Os valores de $r_{a 1 a 2}(0,58$ e 0,61$)$ sugerem que a seleção de fêmeas com base em P240 ou P365 levaria à obtenção de matrizes com maiores probabilidades de permanecerem por mais tempo no rebanho. Resultados semelhantes foram obtidos por TANIDA et al. (1988).

Com base nos valores das estimativas de correlações genéticas entre as características de cresci- mento e reprodução obtidas, sugere-se que, em geral, a seleção de bezerras para reposição de matrizes, com base em maiores pesos aos 240 e 365 dias, levaria à obtenção de progênies mais pesadas a estas idades (considerando os valores de $\mathrm{h}^{2}$ a obtidos para P240 e P365), sem efeitos deletérios no futuro desempenho reprodutivo, no caso das fêmeas, permitindo também a obtenção de fêmeas mais longevas.

Os resultados dos poucos estudos considerando crescimento e reprodução em fêmeas criadas em ambiente tropical são, até certo ponto, contraditórios e, geralmente, indicam antagonismo genético (MARIANTE e ZANCANER, 1985; BARBOSA, 1991). Entretanto, trabalhos mais recentes (MASCIOLI et al., 1999; SILVA et al., 1999) têm sugerido sinergia genética entre crescimento e idade ao primeiro parto. KOOTS et al. (1994b), em extensa revisão sobre parâmetros genéticos de características de bovinos de corte, mostraram valores contraditórios e enfatizaram o pequeno número de estudos relacionando crescimento e reprodução.

Sob outro ponto de vista, observando as estimativas de correlações genéticas $\left(\mathrm{r}_{\mathrm{a} 2 \mathrm{~m} 1}\right)$ entre o efeito materno das características de crescimento (P240 e P365) e o efeito direto das características de reprodução na Tabela 2, todos os valores, com exceção de LONG, apresentaram sentido antagônico à seleção, sugerindo que as matrizes que produzem mais leite apresentam baixo desempenho reprodutivo. Essa tendência pode ser confirmada pelos valores de correlações genéticas iguais a 0,60 e $-0,12$ e fenotípicas iguais a 0,11 e - 0,56 entre IEP1, ER e a característica P240B, mostrados na Tabela 3.

Resultados semelhantes foram obtidos por ALENCAR et al. (1993), com animais da raça Nelore, que notaram aumento de 0,11 dias no intervalo de partos para cada quilo de leite produzido pela matriz; por REGE e FAMULA (1993), trabalhando com animais da raça Hereford, que observaram que as vacas com maior índice de valor genético maternal pariam mais tarde na estação de parição; e por SWANEPOEL e HOOGENBOEZEN (1994b), com a raça Africander, os quais observaram que as vacas que apresentaram menores intervalos de partos desmamaram bezerros mais leves. Sobre este fato, DAVIS et al. (1993) comentaram que as vacas que produzem menos leite podem apresentar melhor habilidade para resistir ao estresse nutricional; anestros pós-parto mais curtos concebendo mais cedo na estação de monta; e, desse modo, melhores desempenhos reprodutivos. DOREN et al. (1986) salientaram 
que, se os produtores selecionarem para maiores pesos ao desmame, o intervalo de partos, em sistemas de produção em pasto, poderá aumentar e o total de peso de bezerro desmamado anualmente, por conseguinte, ser reduzido.

\section{Correlações entre características de reprodução}

As estimativas de correlações genéticas e fenotípicas entre as características de reprodução e produção estudadas são mostradas na Tabela 3. De modo geral, as estimativas foram todas de sentido favorável à seleção, com exceção de IEP1, ER com P240B, já discutido anteriormente.

A estimativa de correlação genética obtida entre IPP e IEP1 apresentou magnitude média e sentido favorável $(0,53)$, sugerindo que a seleção de novilhas sexualmente mais precoces poderia levar a animais mais eficientes em termos de intervalos de parto. Este resultado é similar aos obtidos por MARIANTE e ZANCANER (1985), que também utilizaram dados da Fazenda Bonsucesso, mas com amostra menor, embora HAILE-MARIAM e KASSA-MERSHA (1994) e LÔBO (1998), utilizando metodologia semelhante ao do presente estudo, tenham reportado resultados bem diferentes.

Idade ao primeiro parto e ER mostraram alto valor de correlação genética, com sentido favorável. Provavelmente, esta forte relação entre as características deve-se ao fato de a fórmula de ER incluir IPP. O mesmo aconteceu com IEP1 e ER. As correlações, tanto genética como fenotípica, de IPP com LONG sugerem que novilhas sexualmente mais precoces têm maior probabilidade de permanecer por mais tempo no rebanho, sendo, portanto, mais produtivas.

Os valores e o sentido das estimativas de correlação genética e fenotípica $(0,68$ e 0,23$)$ entre ER e LONG concordam com os resultados obtidos por ARTHUR et al. (1992) e SWANEPOEL e HOOGENBOEZEN (1994a), ou seja, as vacas que permanecem por mais tempo no rebanho são aquelas que apresentam melhores desempenhos reprodutivos durante a vida útil, istoé, menor IPP e menores intervalos de partos, conseqüentemente maiores valores de ER.

\section{Implicações}

As características de crescimento estudadas, $\mathrm{P} 240$ e P365, mostraram variabilidade genética considerável, sugerindo que a seleção de fêmeas com base, principalmente, no peso aos 365 dias levará à obtenção de progênies com mais altos pesos a estas idades, com pequeno ou nenhum efeito negativo sobre a habilida- de materna das fêmeas que se tornarem matrizes. Além disso, as correlações genéticas próximas de zero encontradas entre os referidos pesos e as características de reprodução demonstraram que a seleção para crescimento não traria efeitos deletérios no desempenho reprodutivo das matrizes.

A característica IPP apresentou, comparativamente às outras, coeficiente de $\mathrm{h}^{2}{ }_{\mathrm{a}}$ substancialmente maior, sugerindo a possibilidade de ganho genético mediante seleção, além de apresentar a vantagem de ser observada relativamente cedo na vida da matriz e estar geneticamente correlacionada com grande parte das características estudadas. Muitos criadores têm selecionado por menores idades ao primeiro parto e alcançado progressos consideráveis nesta característica, fazendo com que novilhas Nelore, raça considerada tardia em termos de puberdade, apresentem idade ao primeiro parto por volta dos 24 meses de idade (LOBATO, 1995).

A característica ligada à permanência do animal no rebanho, como esperado, apresentou baixo coeficiente de herdabilidade. Obviamente, característica cuja informação só pode ser obtida tardiamente na vida do animal, como LONG, não pode ser preconizada como critério de seleção direta. Apesar disso, a "Associação Americana de Red Angus" tem calculado DEPs para a probabilidade de a matriz permanecer no rebanho e parir até a idade de 6 anos (SNELLING et al., 1994).

A característica considerada de produção, $\mathrm{P} 240 \mathrm{~B}$, mostrou razoável variabilidade genética. Considerando que o descarte de vacas com base no peso de seus bezerros seja prática comum e importante entre os criadores de gado de corte, mais estudos deste tipo são requeridos, principalmente em animais criados em pasto sob condições tropicais, especialmente no que se refere às relações entre reprodução da matriz e peso ao desmame da progênie.

\section{Conclusões}

O coeficiente de herdabilidade estimado para a característica idade ao primeiro parto indica possibilidade de ganho genético considerável na precocidade sexual das novilhas, com resposta correlacionada favorável nas outras características de reprodução (primeiro intervalo de partos, eficiência reprodutiva e anos de permanência da matriz no rebanho). Os valores das estimativas de correlação genética entre os pesos estudados e as características de reprodução indicam que a seleção de fêmeas para maiores pesos padronizados aos 240 e 365 dias não prejudicaria seu 
desempenho reprodutivo. O descarte de vacas jovens com base no desempenho ponderal à desmama de seu primeiro bezerro levaria à obtenção de matrizes mais eficientes em termos de quilogramas de bezerros desmamados, porém com diminuição do valor genético dos animais para menores intervalos de partos.

\section{Referências Bibliográficas}

ALBUQUERQUE, L.G., FRIES, L.A. Parâmetros genéticos da produção de leite e crescimento do bezerro até a desmama, como característica da vaca. In: REUNIÃO ANUAL DA SOCIEDADE BRASILEIRA DE ZOOTECNIA, 34, Juiz de Fora, 1997. Anais....Juiz de Fora, 1997, v.3, p.22-24.

ALENCAR, M.M., OLIVEIRA, F.T.T., TAMBASCO, A.J. et al. 1993. Desenvolvimento pós-desmama e eficiência reprodutiva pós-parto em gado de corte: influência da produção de leite. R. Soc. Bras. Zootec., 22:1012-1018.

ARTHUR, P.F., MAKARECHIAN, M., BERG, R.T. et al. 1992. Reasons for disposal of cows in a purebred Hereford and two synthetic groups under range conditions. Can. J. Anim. Sci., 72:751-758.

BARBOSA, P.F. 1991. Análise genético-quantitativa de características de crescimento e reprodução em fêmeas da raça Canchim. Ribeirão Preto, SP. FMRP, 1990. 237p. Tese (Doutorado em Ciências Biológicas) - Faculdade de Medicina de Ribeirão Preto/Universidade de São Paulo, 1990.

BEZERRA, L.A.F., DUARTE, F.A.M. Nova fórmula para avaliação da eficiência reprodutiva de vacas. In: REUNIÃO ANUAL DA SOCIEDADE BRASILEIRA DE ZOOTECNIA, 17, 1980, Fortaleza. Anais... Fortaleza: SBZ, 1980, p.169.

BOLDMAN, K.G., KRIESE, L.A., VAN VLECK, L.D. et al. 1993. A manual for use of MTDFREML. Lincoln: Department of Agriculture/Agricultural Research Service. 120p.

DAVIS, G.P., CORBET, N.J., MACKINNON, M.J. et al. 1993. Response in female fertility and calf growth to selection for pregnancy rate in tropical beef cattle. Aust. J. Agric. Res., 44:1509-1521.

DOREN, P.E., LONG, C.R., CARTWRIGHT, T.C. 1986. Factors affecting the relationship between calving interval of cows and weaning weights of calves. J. Anim. Sci., 62:1194-1202.

HAILE-MARIAM, M., KASSA-MERSHA, H. 1994. Genetic and environmental effects on age at first calving and calving interval of naturally bred Boran (Zebu) cows in Ethiopia. Anim. Prod. 58:329-334.

KOOTS, K.R., GIBSON, J.P., SMITH, C. et al. 1994a. Analyses of published genetic parameters estimates for beef production traits. 1 - Heritability. Anim. Breed. Abstr. 62:309-338.

KOOTS, K.R., GIBSON, J.P., WILTON, J.W. 1994b. Analyses of published genetic parameters estimates for beef production traits. 2 - Phenotypic and genetics correlations. Anim. Breed. Abstr., 62:825-853.

LOBATO, J.F.P. Produção e manejo de gado de corte. In: REUNIÃO ANUAL DA SOCIEDADE BRASILEIRA DE ZOOTECNIA, 32, Brasília, 1995. Anais.... Brasilia: SBZ, 1995, p.405-414.

LÔBO, R.N.B. 1998. Genetic parameters for reproductive traits of Zebu cows in the semi-arid region of Brazil. Lvstck. Prod. Sci., 55:245-248.

MARIANTE, A.S., ZANCANER, A. 1985. Crescimento $e$ reprodução em gado Nelore. Visão do criador e do pesquisador. 1.ed. São Paulo: Ed. dos Criadores. 152p.

MASCIOLI, A.S., TALHARI, F.M., ALENCAR, M.M. et al. Correlações genéticas entre características reprodutivas e de crescimento de fêmeas da raça Canchim. In: REUNIÃO ANUAL DA SOCIEDADE BRASILEIRA DE
ZOOTECNIA, 36, Porto Alegre, 1999. Anais....Porto Alegre, 1999, p.156.

MERCADANTE, M.E.Z., LÔBO, R.B. 1997. Estimativas de (co)variâncias e parâmetros genéticos dos efeitos direto e materno de características de crescimento de fêmeas de um rebanho Nelore. R. Bras. Zootec., 26:1124-1133.

MERCADANTE, M.E.Z., LÔBO, R.B., REYES, A. 1995. Parámetros genéticos para características de crecimiento en cebuínos de carne. Arch. Latinoam. Prod. Anim., 3:45-89.

MEYER, K., CARRICK, M.J., DONNELY, B.J.P. 1994. Genetic parameters for milk production of Australian beef cattle cows and weaning weight of their calves. J. Anim. Sci., 72:1155-1165.

MEYER, K., HAMMOND, K., MACKINNON, M.J. et al. 1991. Estimates of covariances between reproduction and growth in Australian beef cattle. J. Anim. Sci., 69:3533-3543.

MEYER, K., HAMMOND, K., PARNELL, P.F. et al. 1990. Estimates of heritability and repeatability for reproductive trait in Australian beef cattle. Lvstck. Prod. Sci., 25:15-30.

MOHIUDDIN, G. 1993. Estimates of genetic and phenotypic parameters of some performance traits in beef cattle. Anim. Breed. Abstr. 61:495-522.

MORRIS, C.A. 1990. Theoretical and realised responses to selection for reproductive traits. In: WORD CONGRESS ON GENETIC APPLIED TO LIVESTOCK PRODUCTION, 4, Edinburgh, Scotland, 1990. Proceedings.... Edinburgh, 1990, v.16, p.309-318.

NOTTER, D.R. 1995. Maximizing fertility in animal breeding programs. In: CONGRESSO BRASILEIRO DE REPRODUÇÃO ANIMAL, 11, 1995, Belo Horizonte (sem pag.).

OLIVEIRA, H.N. 1995. Análise genético-quantitativa da curva de crescimento de fêmeas da raça Guzerá. Ribeirão Preto, SP: FMRP, 1995. 73p. Tese (Doutorado em Ciências Biológicas) - Faculdade de Medicina de Ribeirão Preto/Universidade de São Paulo, 1995.

REGE, J.E.O., FAMULA, T.R. 1993. Factors affecting calving date and its relationship with production traits of Hereford dams. Anim. Prod., 57:385-395.

SMITH, B.A., BRINKS, J.S. 1989. Estimation of genetic parameters among reproductive and growth traits in yearling heifers. J. Anim. Sci., 67:2886-2891.

SNELLING, W.M., GOLDEN, B.L., BOURDON, R.M. An EPD for stayability of beef cows. In: WORD CONGRESS ON GENETIC APPLIED TO LIVESTOCK PRODUCTION, 5, 1994, Guelph, Canada. Proceedings.... Guelph, 1994, v.17, p.169-172.

SILVA, A.M., AlENCAR, M.M., OLIVEIRA, M.C. et al. Correlações genéticas entre peso de machos e características reprodutivas e de crescimento de fêmeas, na raça Canchim. In: REUNIÃO ANUAL DA SOCIEDADE BRASILEIRA DE ZOOTECNIA, 36, 1999, Porto Alegre. Anais....Porto Alegre: SBZ, 1999, p.156.

SWANEPOEL, F.J.C., HOOGENBOEZEM, J.M. Longevity in beef cattle. In: AUSTRALIAN SOCIETY ANIMAL PRODUCTION, Perth, Austrália, 1994. Proceedings.... Perth, 1994a, v.20, p.343.

SWANEPOEL, F.J.C., HOOGENBOEZEM, J.M. Interrelationship among cow size, lifetime cow fertility, milk production and pre-weaning calf growth in sub-tropically adapted beef cattle. In: AUSTRALIAN SOCIETY OF ANIMAL PRODUCTION, Perth, Australia, 1994. Proceedings.... Perth, 1994b, v. 20, p.78-80.

TANIDA, H., HOHENBOKEN, W.D., DENISE, S.K. 1988. Genetic aspects of longevity in Angus and Hereford cows. J. Anim. Sci., 66:640-647.

Recebido em: 12/04/1999 Aceito em: 30/11/1999 